\title{
The almost monoenergetic ion event on 19 October 2009: SEPT/STEREO observations
}

\author{
A. Klassen ${ }^{1}$, R. Gómez-Herrero ${ }^{1}$, R. Müller-Mellin ${ }^{1}$, B. Heber ${ }^{1}$, R. F. Wimmer-Schweingruber ${ }^{1}$, \\ A. Opitz ${ }^{2}$, and J.-A. Sauvaud ${ }^{2}$ \\ ${ }^{1}$ Institut für Experimentelle und Angewandte Physik, Universität Kiel, 24118 Kiel, Germany \\ e-mail: klassen@physik.uni-kiel.de \\ 2 Centre d'Étude Spatiale des Rayonnements, Université de Toulouse (UPS), France
}

Received 30 March 2010 / Accepted 31 January 2011

\begin{abstract}
The Solar Electron and Proton Telescope (SEPT) on board the twin spacecraft STEREO A and B measures electrons and ions in the energy range from 60 to above $400 \mathrm{keV}$ with an energy resolution of 10\%. On 19 October 2009, when STEREO-B was already $1.03 \mathrm{AU}$ away from the Earth, a strong and prolonged almost monoenergetic ion event has been observed with the SEPT instrument. The event lasted 27 min and its energy spectrum contained a strong narrow peak at $235 \mathrm{keV}$ with a relative full width at half maximum of 0.35 . The event occurred during a period of slow solar wind in front of a weak ion increase associated with a distant corotating interaction region (CIR). Previously similar events containing spectral peaks were detected in the vicinity of the Earth's magnetosphere using observations on Interball-1 and on both STEREO A \& B spacecraft.

We present evidence that the narrow spectral peak is caused by a quasi-monoenergetic ion beam and suggest that the particles were accelerated at a distant CIR or CIR shock. We discuss the possible mechanisms that could be responsible for accelerating these ions: the shock drift acceleration, the surfatron mechanism and the acceleration in a large-scale electrostatic field.
\end{abstract}

Key words. interplanetary medium - acceleration of particles - shock waves

\section{Introduction}

There are three main sources of energetic ions in the range of $100-2000 \mathrm{keV}$ observed at distances of $\sim 1$ AU from the Sun: the Sun itself (ions accelerated in flares and coronal shocks), interplanetary (IP) shocks driven by coronal mass ejections (CMEs) or by corotating interaction regions (CIRs), and the Earth's bowshock/magnetosphere. The particles associated with IP shocks, which are of interest in this paper, were usually interpreted as being accelerated via shock drift acceleration (SDA) or Fermi acceleration mechanisms (e.g. Scholer et al. 1981; Burgess 2007; Desai \& Burgess 2008, and references therein). The mechanisms by which the IP shock accelerates particles are of physical interest, and the spectra of these accelerated particles can be used as a diagnostic tool for acceleration processes acting in the interplanetary medium. Both SDA and Fermi mechanisms predict a power-law-like energy spectrum with a negative slope without any narrow spectral structures. However, in the last decade a few investigations revealing narrow peaks in ion energy spectra have been reported:

1. Lutsenko \& Kudela (1999) first reported observations of 1,2 or 3 narrow peaks $\left(\triangle E / E_{\max } \simeq 0.15 \div 0.30\right)$ in spectra of upstream ion events observed close to Earth's bow-shock. Similar narrow spectral peaks with $\left(\triangle E / E_{\max } \simeq 0.2 \div 0.7\right)$ were observed with SEPT/STEREO not only close to the bow-shock but also far away from the Earth at distances up to $1900 R_{\mathrm{E}}$ (Müller-Mellin et al. 2008a; Klassen et al. 2009). It was suggested that the multiple energy peaks were caused by quasi monoenergetic beams of $\mathrm{H}^{+}, \mathrm{He}^{++}$and $\mathrm{CNO}^{(5-6)+}$ accelerated at the bow-shock or in the magnetosheath/magnetopause (Klassen et al. 2009). These ion events have been called "almost monoenergetic ions" (AMI) and were explained as ions being accelerated in a large-scale electrostatic field to energies proportional to their charges $Q$ as proposed by Lutsenko \& Kudela (1999).

Anderson (1981) and Anagnostopoulos et al. (2000) also reported observations of upstream events whose energy spectra contained maxima near $100 \mathrm{keV}$. These maxima were explained as special propagation effects and as reflecting different stages in the leakage process of magnetospheric ions towards the upstream region and not as a result of a specific acceleration mechanism.

2. Peculiar energy spectra exhibiting relative broad maxima $\left(\triangle E / E_{\max } \sim 1\right)$ at energies about $0.25-1.5 \mathrm{MeV}$ observed near IP shock fronts driven by CMEs were observed at the Ulysses and ACE spacecraft (Simnett 2003). It was suggested that these spectra could be explained by the surfatron mechanism acting at a fast magnetosonic shock wave (Simnett et al. 2005).

3. The first AMI event in association with an IP shock driven by a CIR was detected with SEPT/STEREO-A. The event spectrum exhibited a narrow spectral peak at $165 \mathrm{keV}$ with $\Delta E / E_{\max } \simeq 0.3$ (Klassen et al. 2009).

Monoenergetic electron and ion beams are very common phenomena in the Earth's aurora region. These particles occur in association with so-called inverted V-structures that are explained as acceleration in electrostatic potential drops in regions of downward/upward currents (e.g. Paschmann et al. 2002).

In this paper we present observations of a strong AMI event detected with STEREO-B (STB) on 19 October 2009 that is associated with the passage of the outskirts of a distant CIR. The main difference from previously observed AMI events is its 
long duration (27 $\mathrm{min}$ ), the high intensity of the spectral peak in comparison to the background spectrum and its detection very far away from the Earth (1.03 AU).

\section{Instruments and data selection}

The observations were carried out with the Solar Electron and Proton Telescopes (SEPT) from the Solar Energetic Particle (SEP) suite of the STEREO IMPACT investigation. SEPT consists of two dual double-ended magnet/foil particle telescopes that separate and measure electrons in the energy range 30$400 \mathrm{keV}$ and ions from 60 to $7000 \mathrm{keV}$ (Müller-Mellin et al. 2008 b). The energy range is divided into 32 logarithmically spaced channels with an energy resolution of $\simeq 10 \%$ in the range $\leq 1200 \mathrm{keV}$ where the AMI events were observed.

Anisotropy information on the non-spinning spacecraft is provided by two separate telescopes: SEPT-E looking in the ecliptic plane along the Parker spiral magnetic field (i.e. $45^{\circ}$ west of the spacecraft-Sun line) both towards and away from the Sun, and SEPT-NS looking perpendicular to the ecliptic plane towards north and south. The dual set-up refers to two adjacent sensor apertures for each of the four view directions: one for protons, one for electrons. The double-ended set-up refers to the detector stack with view cones in two opposite directions: one side (electron side) is covered by a thin foil, the other side (proton side) is surrounded by a magnet. The foil leaves the electron spectrum essentially unchanged but stops protons up to $400 \mathrm{keV}$. The magnet sweeps away electrons but lets ions pass. The geometry factor for each electron and proton telescope is $0.13 \mathrm{~cm}^{2} \mathrm{sr}$ and $0.17 \mathrm{~cm}^{2} \mathrm{sr}$, respectively. The SEPT time resolution is $1 \mathrm{~min}$ and the field of view (FOV) is $52^{\circ}$. Note that SEPT measures the total energy of ions, but not its nuclear charge and thus is not capable of elemental resolution. Hence, the SEPT spectra are presented on a total energy scale. Complementary magnetic field, solar wind data, and EUVI images were used from MAG (Acuña et al. 2008), PLASTIC (Galvin et al. 2008) and SWEA (Sauvaud et al. 2008), and the SECCHI-EUVI (Howard et al. 2008) instruments aboard STEREO-B.

\section{Observations}

During three years of operation SEPT detected three distinct almost monoenergetic ion (AMI) events of interplanetary origin, two of which were associated with the passage of in-situ detected CIR forward shocks (Klassen et al. 2009). The AMI event detected on 19 October 2009 and discussed here was not associated with an in-situ IP shock passage or any solar activity.

Figure 1 displays in panel (f) the omnidirectional dynamic energy spectrum from 16 to 22 October 2009. The AMI event (arrow) occurs at the onset of a weak ion intensity increase lasting a few days at energies $\leq 300 \mathrm{keV}$ with a spectral index around -3 . No electron enhancements in the SEPT energy range were detected. In panel (e) the SWEA suprathermal electron pitchangle distribution shows an enhancement of the $250 \mathrm{eV}$ electrons after the AMI event.

Panel (d) presents the solar wind speed, the proton density, and the temperature of the plasma during the same time interval. The time of the AMI event is marked by the vertical line. Evidently it was observed during a period of slow solar wind $\left(V_{\mathrm{sw}}=280 \mathrm{~km} \mathrm{~s}^{-1}\right)$.

Panel (a) shows on an enlarged time scale the colour-coded dynamic energy spectrum of ions between $80 \mathrm{keV}$ and $2 \mathrm{MeV}$. The intensity of protons around $200 \mathrm{keV}$ increased and decreased abruptly at 0122 and 0149 UT and showed a maximum
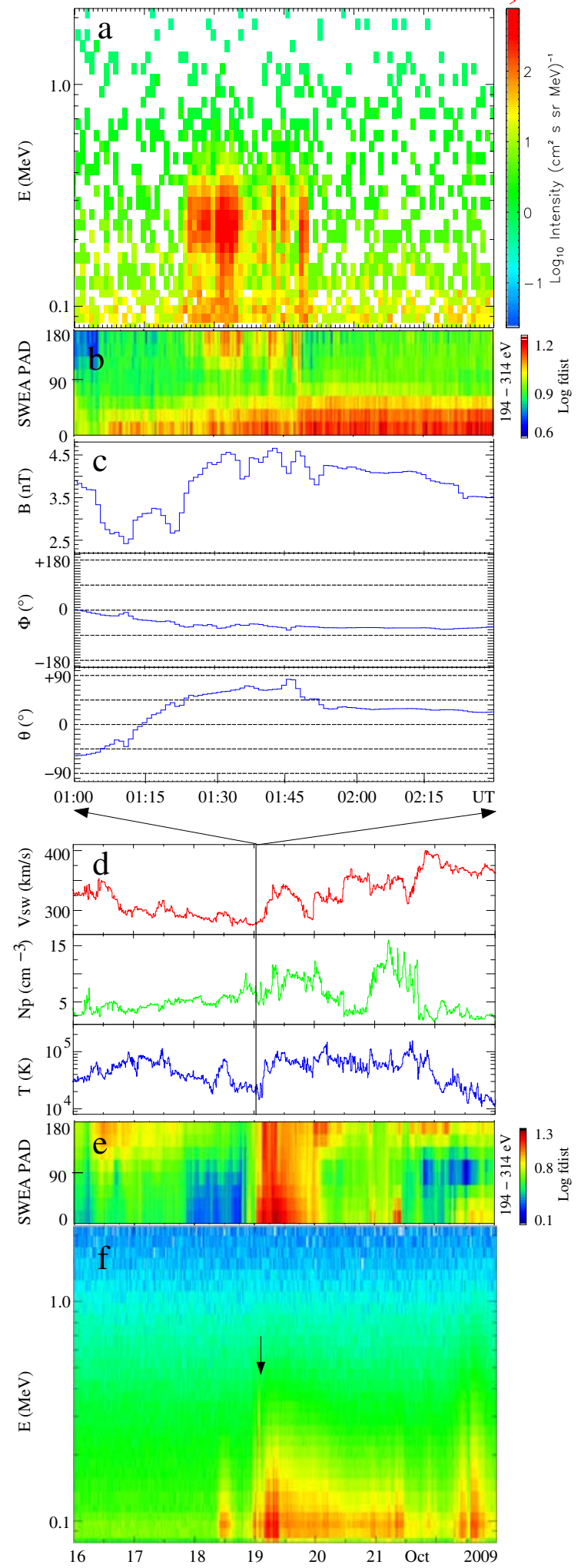

Fig. 1. Panel a) dynamic ion energy spectrum of the AMI event in the energy range $0.08-2.2 \mathrm{MeV}$ observed with SEPT-B/antiSun telescope on 2009-10-19. Panel b) and e) SWEA suprathermal electron pitchangle distribution (PAD) at 194-314 eV. Panel c) IP magnetic field components in spherical coordinates $(|B|, \phi$, and $\theta$ during the event. Panel d)-f) extended time interval from 16 to 22 October 2009. Panel d) solar wind speed, density and proton temperature variations. The vertical line indicates the event time. Panel e) the same as in panel b). Panel f) omnidirectional ion spectrogram showing the AMI event (arrow) in front of a weak particle intensity increase lasting a few days at energies below $300 \mathrm{keV}$. 
at $235 \pm 25 \mathrm{keV}$ for the telescope looking in the anti-Sun direction. A similar structure was detected in the telescope looking towards north (not shown). The two other telescopes looking towards the Sun and south show no increases at all. In addition, the energy peak was stable for the whole event duration, showing no velocity dispersion. The absence of velocity dispersion means that the particle intensity at all energies increased simultaneously. Finally, the whole AMI structure and its peak also do not show drift to higher or lower energies (in Fig. 1a). That usually occurs if a flux tube filled with particles is convected over the spacecraft. Furthermore the AMI event was not accompanied by any electron enhancements at energies between $35-400 \mathrm{keV}$. On the other hand, an additional population of lower energy electrons in the energy range of $0.1-2 \mathrm{keV}$ appeared during the AMI event. The pitch-angle distribution (PAD) of $194-314 \mathrm{eV}$ is shown in Fig. 1b. These electrons counter-stream $\left(\mathrm{PA}=180^{\circ}\right)$ along the magnetic field line (i.e. from the anti-Sun/north direction), while the suprathermal electron strahl from the Sun is flowing parallel $\left(\mathrm{PA}=0^{\circ}\right)$, pointing out that the spacecraft is in the away sector.

Panel (c) shows on the same enlarged time scale the magnetic field magnitude $(\simeq 4.5 \mathrm{nT})$ and its angles $(\phi$ and $\theta)$ in spherical coordinates. The azimuthal angle $(\phi)$ slightly rotates before the event from $-20^{\circ}$ to $-50^{\circ}$, but the latitudinal angle $(\theta)$ strongly rotates from about $-45^{\circ}$ towards $+45^{\circ}$. During the event the $(\phi)$-angle stays almost stable around $-50^{\circ}$, but $(\theta)$ changes between $+45^{\circ}$ and $+80^{\circ}$.

The solar wind conditions were very quiet during the AMI event: solar wind speed $V_{\mathrm{sw}}=280 \mathrm{~km} \mathrm{~s}^{-1}$, density $\simeq 5 \mathrm{~cm}^{-3}$, and proton temperature $T_{\mathrm{p}} \simeq 2 \times 10^{4} \mathrm{~K}$. Some hours after the event $T_{\mathrm{p}}$ and $V_{\text {sw }}$ increased up to $\simeq 1 \times 10^{5} \mathrm{~K}$ and $340 \mathrm{~km} \mathrm{~s}^{-1}$, respectively (Fig. 1d). These small increases together with counter-streaming electrons in spite of slow solar wind speed are indicative of a passage of the outskirts of a distant high-speed solar wind stream.

Evidently the ions, predominantly protons, stream along the IP magnetic field from the anti-Sun/north direction and were detected only in two telescopes looking in these directions. This agrees with the new counter-streaming electron population detected by SWEA. The appearence of the AMIs and the suprathermal electrons is correlated with small variations in magnetic field strength, which occur when the $\theta$-angle changes between $+45^{\circ}$ and $+80^{\circ}$ while the $(\phi)$-angle stays around $-50^{\circ}$.

Figure 2 (top) presents the averaged energy spectra obtained at 0125-0133 UT (first, top spectrum) and at 0136-0150 UT (second one) showing a strong peak at $235 \mathrm{keV}$ (anti-Sun telescope, blue) and for comparison the spectrum without a peak from the opposite direction (Sun telescope, black). Both AMI spectra are different in intensity and slightly in FWHM, but they both peak at $235 \mathrm{keV}$. The spectral shape around the peak is relatively steep. Indeed, the flanks of the peak can be roughly approximated by a power-law. In this case, the negative slope has a $\gamma=-6.1$, and the positive a $\gamma=+4.7$. In comparison the $\gamma$ of unaffected telescopes (SEPT/Sun \& SEPT/south) are about -2.5 .

Time profiles at $235 \mathrm{keV}$ reveal a strong anisotropy with a pronounced 250-fold intensity increase from the anti-Sun and north directions and without any increases in the other two telescopes (Fig. 2 (middle panel)). Furthermore, they contain a sequence of intensity spikes with a duration of 1-2 min especially after 0140 UT. These strong intensity variations could require a bursty acceleration process or they could be caused by propagation effects such as sudden break-offs in the magnetic connection to the source. However, only the intensity spike at 0148 UT was correlated with the small variation in the $\theta$-angle. Table 1 summarizes the main properties of the observed AMI event.
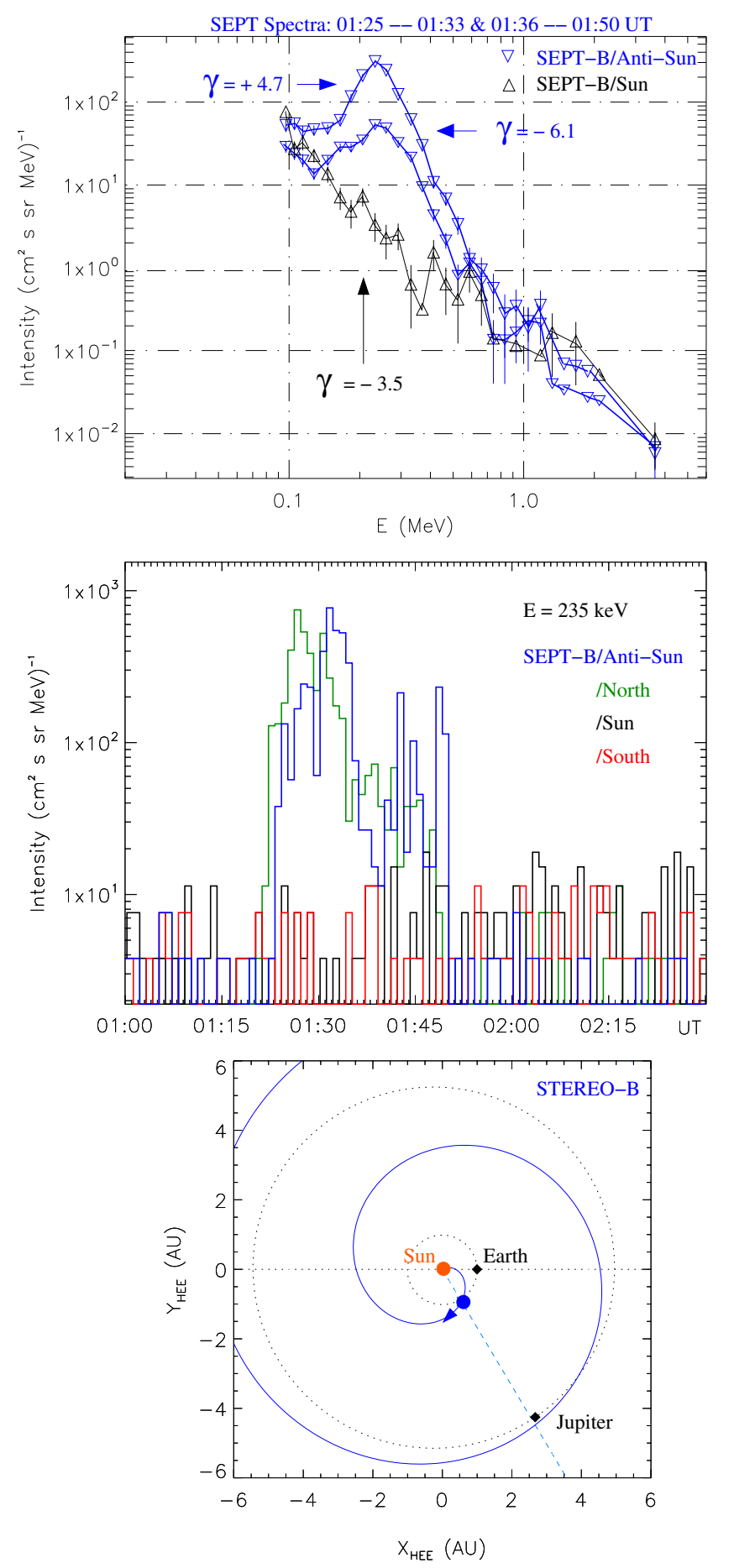

Fig. 2. Top, two averaged ion energy spectra obtained at 0125-0133 UT (the top one, blue) and at 0136-0150 UT (the second one, blue) with a peak at $235 \mathrm{keV}$ from SEPT/anti-Sun and without a peak from the oppositely directed SEPT/Sun (black) telescopes. Middle, time profiles at $235 \mathrm{keV}$ of all four telescopes. The burst is detected from the antiSun and north directions only. Bottom, the position of STEREO-B (blue filled circle), the nominal Parker spiral and the magnetic field ecliptic component during the event (blue arrow). The Earth and Jupiter positions are shown by black diamonds.

Figure 2 (bottom) represents the position of STEREO-B (blue) in relation to the Sun and Jupiter. The blue arrow and the blue line show the STEREO-B ecliptic IMF component during the event and the nominal Parker spiral according to the solar wind speed of $280 \mathrm{~km} \mathrm{~s}^{-1}$ as measured during the AMI event, 
Table 1. Event parameters for almost monoenergetic ions.

\begin{tabular}{lc}
\hline \hline Peak energy $\left(E_{\max }\right), \mathrm{keV}$ & $235 \pm 25$ \\
$-F W H M(\triangle E), \mathrm{keV}$ & 80 \\
$-\triangle E / E_{\max }$ & 0.35 \\
Duration, $\min$ & 27 \\
Peak intensity, $\left(\mathrm{cm}^{2} \mathrm{~s} \mathrm{sr} \mathrm{MeV}\right)^{-1}:$ & \\
-Anti-Sun telescope & 770 \\
- North telescope & 745 \\
Pre-event intensity at $235 \mathrm{keV}$ & 3 \\
\hline
\end{tabular}

respectively. In this ideal case the spacecraft is well connected to Jupiter with a very long path length of 20 AU. However, no relativistic electrons were detected, although Jupiter is a strong source of relativistic electrons. Magnetic connections to other planets are very poor and are not shown.

To summarize the observational results: the SEPT instrument on board STEREO-B observed on 19 October 2009 an intense long-lasting AMI event during slow solar wind conditions. We found that

1. the energy spectrum contains a narrow peak at $E_{\max }=$ $235 \mathrm{keV}$ with a $F W H M$ of $\triangle E=80 \mathrm{keV}$ and $\triangle E / E_{\max }=$ 0.35 . No velocity dispersion was observed throughout the event;

2. the event duration was $27 \mathrm{~min}$ and the time profiles show strong intensity variations;

3. the anisotropic ions stream along the IP magnetic field from the anti-Sun/north direction and were detected only in corresponding telescopes in agreement with an appearing counterstreaming suprathermal electron population of $0.1-2 \mathrm{keV}$;

4. the spectral index around the spectral peak is very steep: up to +4.7 and -6.8 for the positive and negative slope, respectively. That is very different compared with usual CIR spectra, which are flat at low energies with $\gamma \simeq-1.5 \ldots-3$ (e.g. Gómez-Herrero et al. 2009);

5. there was no associated solar activity and the spacecraft was not magnetically connected with the Earth's magnetosphere (Fig. 2). Furthermore, the ions arrived at STB from the antiEarth and anti-Sun direction, implying that neither the Sun nor the Earth were the sources of the AMI event;

6. Although in an ideal case, according to the instantaneous solar wind speed, the Parker spiral connected STEREO-B with Jupiter, the lack of relativistic Jovian electrons means that Jupiter was likely not the source of the AMI event.

\section{Discussion}

\subsection{Possible source of $A M l$ event}

Generally, there are three possible sources for energetic ions: solar activity, Earth's magnetosphere, and CIRs. The solar activity could be excluded as a source because no flares, CMEs or type III radio bursts indicating solar acceleration processes were observed during or some hours before the event. This was ascertained by inspection of SWAVES radio spectrograms, EUVI, COR-1, 2 and Heliospheric Imager data at STEREO, and by using other space- and ground-based resources. STEREO-B was 1.03 AU away and magnetically not connected with the Earth (Fig. 2 bottom). Furthermore the ions were encountered from the anti-Sun and anti-Earth direction. Therefore, the Sun and the Earth's magnetosphere are not the source of the observed event.

Although STEREO-B was apparently magnetically connected to Jupiter, it is unlikely that the AMI really originated

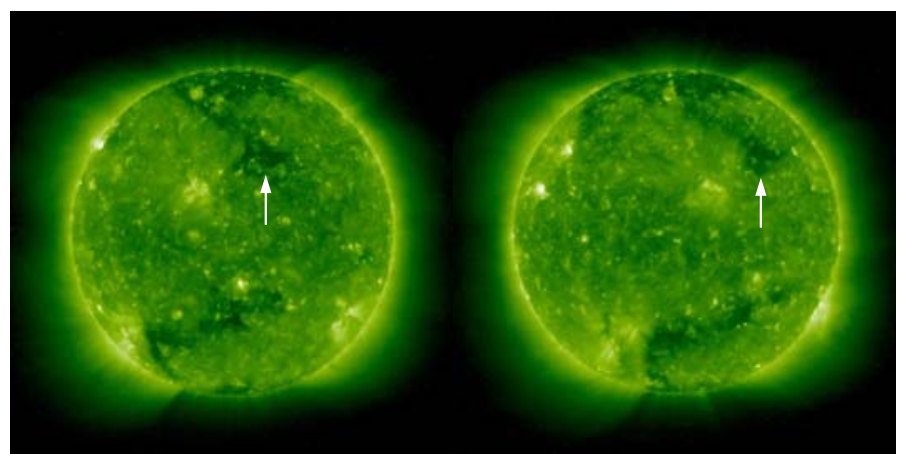

Fig. 3. STEREO-B/EUVI $195 \AA$ A images on 17 and 19 October 2009 showing a small coronal hole in the northwest quadrant, which could be responsible for the high-speed stream and the CIR near STEREO-B on 19 October 2009.

there, because the Parker spiral is not only drawn by this instantaneous $280 \mathrm{~km} \mathrm{~s}^{-1}$ solar wind plasma parcel as observed in-situ during the event. If we take an average speed of $>300 \mathrm{~km} \mathrm{~s}^{-1}$ as measured for a few days before the event, the connection to Jupiter is totally lost. Additionally the remotest ion bursts of Jovian origin observed in the past were detected at distances up to 0.6 AU from Jupiter only (e.g. Krimigis 1992) and they were never detected at the Earth's orbit. Note that in our case the propagation path Jupiter-STEREO-B was 20 AU. The spiky time profile of the AMI event and its occurrence when the magnetic field $\theta$ component turns towards the north and the absence of relativistic electrons also contradict a Jovian origin.

Therefore we suggest that a distant high-speed solar wind stream and an associated CIR/shock occurring northwards and farther away from the spacecraft could be responsible for the accelerated ions. This suggestion is supported by the following observations. Firstly, a few hours after the AMI event the STEREO-B spacecraft entered a region with slightly enhanced solar wind speed $\left(V_{\mathrm{sw}}\right)$ and proton temperature $T_{\mathrm{p}}$ as observed with PLASTIC/STB. The $V_{\text {sw }}$ increased from 280 to $340 \mathrm{~km} \mathrm{~s}^{-1}$ and $T_{\mathrm{p}}$ from $2 \times 10^{4} \mathrm{~K}$ to $1 \times 10^{5} \mathrm{~K}$ (Fig. 1d). Furthermore a CIRlike ion intensity increase in the range $\leq 300 \mathrm{keV}$ is observed after the AMI event as described in Sect. 3 (Fig. 1f). The appearence of counter-streaming suprathermal electrons strongly supports the idea of its origin in conjunction with a CIR, as was observed by Lavraud et al. (2010). These low-energy electrons may be accelerated or/and reflected at the CIR.

Secondly, Fig. 3 shows EUVI images on 17 and 19 October 2009, revealing a small coronal hole in the Sun's northwest quadrant, which could be an appropriate source of the highspeed stream and associated CIR/shock near to STEREO-B on 19 October. The coronal hole was relatively small with sharp contours. Therefore it could be expected that the outflowing high-speed stream and the CIR was narrow and only the outskirts of the stream/CIR were convected over the spacecraft, followed by a small increase of solar wind speed.

Thirdly, a few previously observed short AMI events were detected in association with CIRs and passage of CIR forward shocks, e.g. Klassen et al. (2009).

Based on all these facts we suggest that the AMI event and the following ion intensity increase originated and were accelerated at a distant CIR/shock, the outskirts of which swept over STEREO-B some hours later. During the event the spacecraft experienced a remote magnetic connection to the CIR/shock located farther away and to the north from the spacecraft. A possible configuration of STB position with respect to the presumed 


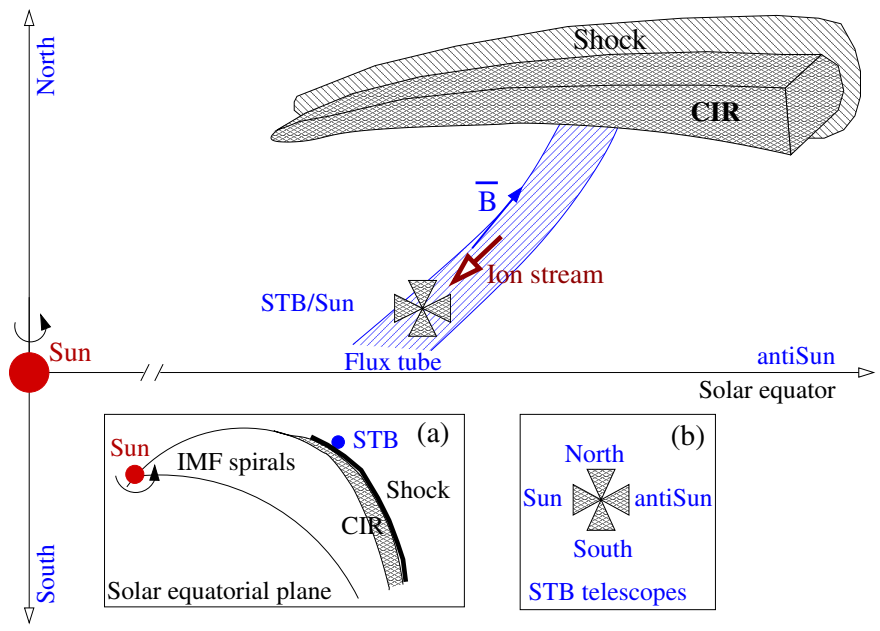

Fig. 4. Schematic diagram of the STEREO-B position with respect to the supposed CIR/CIR shock. The magnetic flux tube is convected over STB and is filled with nearly scatter-free ions streaming along the magnetic field from the CIR towards STB. The ions are only detected in two telescopes looking towards anti-Sun and north. Inserts: a) Parker spirals and the CIR region in projection on the solar equatorial plane, b) fields of view of all four SEPT telescopes.

CIR and CIR shock is presented in Fig. 4. A magnetic flux tube convected over the spacecraft is connecting with the CIR/shock and is filled with nearly scatter-free ions streaming along the IP magnetic field from the CIR towards STB. The sketch gives an idea why the particles streaming from a distant source were detected only in telescopes looking towards anti-Sun and north during times when the flux tube is convected over the STB.

The Heliospheric Imager (HI/STA) did not detect any density structures like a CIR or CME close to STB. Very probably the expected CIR was not visible by HI owing to the large separation angle of $121^{\circ}$ between STA and STB, i.e. STB was significantly behind the Thomson surface (the surface of maximum scatter). Additionally, the expected structure was partly occulted by a CME propagating in the STA/Earth direction (Davis \& Davies, priv. comm.).

\subsection{Acceleration mechanisms}

Observations of narrow spectral features in ion spectra associated with IP shocks are relatively rare, but not unique (Simnett et al. 2005; Klassen et al. 2009). For instance, during three years of the STEREO mission we detected only three of these events. One of them is described in Klassen et al. (2009). Mechanisms responsible for AMI generation are not yet really understood.

Principally there are only three main mechanisms that are able to explain energy spectra containing features like maxima. Firstly, the shock drift mechanism (SDA). Under some specific conditions the mechanism predicts a broad maximum around $100 \mathrm{keV}$ in ion energy spectra (e.g. Figs. 4, 5 in Decker \& Vlahos 1986, and references therein). But the authors note that "...the upstream spectrum folds over smoothly with decreasing energy...". Therefore the predicted width of the spectral maximum $\left(\triangle E / E_{\max }>1\right)$ is too broad to explain the detected narrow energy peak with $\triangle E / E_{\max }>0.35$.

Secondly, the $\boldsymbol{V}_{\mathrm{p}} \times \boldsymbol{B}$ acceleration (surfatron) process (Sagdeev \& Shapiro 1973; Ohsawa \& Sakai 1987). Here $\boldsymbol{V}_{\mathrm{p}}$ is the wave phase velocity, $\boldsymbol{B}$ the magnetic field. For a shock, $\boldsymbol{V}_{\mathrm{p}}$ is just the shock velocity with respect to the upstream plasma (Decker 1988). It was shown that quasi-perpendicular shocks accelerate trapped ions in the direction parallel to the wave front with a peak in energy distribution. Simnett et al. (2005) argued that the surfatron mechanism can explain spectral maxima with $\triangle E / E_{\max } \geq 1$ as observed at the Ulysses and ACE spacecraft during passages of shock fronts. Again, the predicted spectral maximum is considerably broader than the observed one.

Thirdly, since the discussed spectrum is very alike the AMI events observed in the vicinity to the Earth's magnetosphere, we suggest that the mechanism could be the same as the one proposed by Lutsenko \& Kudela (1999) for magnetospheric AMIs. In this case the acceleration of monoenergetic ions occur in a large-scale potential electrostatic field $\boldsymbol{E}_{\text {stat }}$. This $\boldsymbol{E}_{\text {stat }}$ accelerates all species of plasma ions to the same energy of $E=$ $E_{\text {stat }} D Q$, were $D=$ source size, $Q=$ ion charge. Consequently, this kind of acceleration naturally explains the narrowness of the observed AMI energy peak $\left(\triangle E / E_{\max }=0.35\right)$ and thus the very steep spectral shape at both sides of the peak maximum ( $\gamma$ up to +4.7 and -6.8). Additionally, it explains the lack of high-energy electrons. Indeed, if $\boldsymbol{E}_{\text {stat }}$ in the source is parallel to the magnetic field $\boldsymbol{B}$, the electrons should be accelerated in the opposite direction than ions. If $\boldsymbol{E}_{\text {stat }}$ is perpendicular to $\boldsymbol{B}$, the electrons will be swept out of the source because of their much smaller gyroradii and because of the $\boldsymbol{E}_{\text {stat }} \times \boldsymbol{B}$ drift. In this case the gyroradius of ions (electrons) should be greater (smaller) than the source size $(D)$. Typical gyroradius values of ions and electrons in the $0.1-1.0 \mathrm{MeV}$ range are about $10000 \mathrm{~km}$ and $100 \mathrm{~km}$, respectively. Consequently the source size could be in the same range. Finally, this model predicts the acceleration of all ion species to the same energy. The lack of other spectral peaks, e.g. of $\mathrm{He}^{++}$, which should appear around $470 \mathrm{keV}$, at twice the energy compared with protons, is not wholly clear. Possibly it is caused by the low solar wind $\mathrm{He}^{++}$abundance in this time. Indeed, during solar cycle minima and the times of low solar wind speed the ratio of $\mathrm{He}^{++}$to protons is strongly decreased to $1 \%$ (e.g. Aellig et al. 2001). Thus the expected $\mathrm{He}^{++}$intensity would be comparable with the background level. Moreover, it would be hidden by the flank of the AMI proton peak.

The acceleration of monoenergetic ions through an electrostatic field more adequately explains the main feature of AMIs compared with other mechanisms, namely the narrowness of the energy peak. One important question is still open: how are these electrostatic fields generated and maintained in interplanetary sources in general and in CIRs in particular? To understand this we need more observational events, especially when the spacecraft crosses the acceleration source.

\section{Conclusion}

We presented observations of a long-lasting, almost monoenergetic ion event whose energy spectrum contains a strong narrow spectral peak at $235 \mathrm{keV}$ with a width of $\Delta E / E_{\max }=0.35$. The event occurred during slow solar wind speed, a few hours before a weak CIR ion increase in conjunction with counter-streaming suprathermal electrons. The anisotropic monoenergetic beam arrived at the STEREO-B spacecraft from the anti-Sun and antiEarth direction, excluding the Sun and the Earth as the source for the almost monoenergetic ions.

We suggest that the monoenergetic ions, predominantly protons, were accelerated by an electrostatic field to energies proportional to their charge at a distant CIR or CIR shock. Other mechanisms such as shock drift acceleration or the surfatron process may also be responsible for the spectrum with a maximum, but apparently they are unable to explain the narrowness of the detected spectral peak. 
Acknowledgements. The authors thank Ch. Davis \& J. Davies for providing the HI data and discussing CIR structures. Andrea Opitz acknowledges discussions with B. Lavraud, N. Andre, B. Lembege, and Z. Yang. The authors thank two anonymous referees for their comments and suggestions. The STEREO/SEPT project is supported under grant 50 OC 0902 by the German Bundesministerium für Wirtschaft through the Deutsches Zentrum für Luft- und Raumfahrt (DLR).

\section{References}

Acuña, M. N., Curtis, D., Scheifele, J. L., et al. 2008, Space Sci. Rev., 136, 203 Aellig, M. R., Lazarus, A. J., \& Steinberg, J. T. 2001, in Solar and Galactic composition, ed. by R. F. Wimmer-Schweingruber, 89

Anagnostopoulos, G. C., Paschalidis, N., \& Littas, N. 2000, Ann. Geophys., 18, 28

Anderson, K. A. 1981, J. Geophys. Res., 86, 4445

Burgess, D. 2007, in Lect. Notes in Phys., ed. K.-L. Klein \& A.L. MacKinnon (Berlin: Springer Verlag), 725, 161

Galvin, A. B., Kistler, L. M., Popecki, M. A., et al. 2008, Space Sci. Rev., 136, 437

Gómez-Herrero, R., Klassen, A., Müller-Mellin, R., et al. 2009, J. Geophys. Res., 114, A05101
Howard, R. A., Moses, J. D., Vourlidas, A., et al. 2008, Space Sci. Rev., 136, 67 Decker, R. B. 1988, Space Sci. Rev., 48, 195

Decker, R. B., \& Vlahos, L. 1986, J. Geophys. Res., 91, 13349

Desai, M. I., \& Burgess, D. 2008, J. Geophys. Res., 113, A00B06

Klassen, A., Gómez-Herrero, R., Müller-Mellin, R., et al. 2009, Ann. Geophys., 27, 2077

Krimigis, S. M. 1992, Space Sci. Rev., 59, 167

Lavraud, B., Opitz, A., Gosling, J. T., et al. 2010, Ann. Geophys., 28, 233

Lutsenko, V. N., \& Kudela, K. 1999, Geophys. Res. Lett., 26, 413

Müller-Mellin, R., Gómez-Herrero, R., Böttcher, S., et al. 2008a, Proc. of the 30th ICRC, Mexico, 1, 371

Müller-Mellin, R., Böttcher, S., Falenski, J., et al. 2008b, Space Sci. Rev., 136, 363

Ohsawa, Y., \& Sakai, J.-I. 1987, ApJ, 313, 440

Paschmann, G., Haaland, S., \& Treumann, R. 2002, Auroral Plasma Physics, Space Sci. Rev., 103, Chap. 7

Sagdeev, R. Z., \& Shapiro, V. D. 1973, JETP Lett., 17, 279

Sauvaud, J.-A., Larson, D., Aoustin, C., et al. 2008, Space Sci. Rev., 136, 227

Scholer, M., Hovestadt, D., Ipavich, F. M., \& Gloeckler, G. 1981, J. Geophys. Res., 86, 9040

Simnett, G. M. 2003, Sol. Phys., 213, 387

Simnett, G. M., Sakai, J.-I., \& Forsyth, R. J. 2005, A\&A, 440, 759 